
\title{
$\begin{array}{ll}\text { Research Square } & \begin{array}{l}\text { They should not be considered conclusive, used to inform clinical practice, } \\ \text { or referenced by the media as validated information. }\end{array}\end{array}$ \\ PGGHG and ODF3B as New Tumor Suppressor Genes in Renal Cell Carcinoma
}

elham mohammadisoleimani ( $\square$ elham.mohamadi.soleimani@gmail.com )

Fasa University of Medical Science

\section{Zahra Firoozi}

Fasa University of Medical Science

Hamed Haghi-Aminjan

Ardebil University of Medical Sciences

Mohammad Mehdi Naghizadeh

Fasa University of Medical Science

Shahryar Zeighami

Shiraz University of Medical Sciences

\section{Ali Ariafar}

Shiraz University of Medical Sciences

Hosein Mansoori

Fasa University of Medical Science

Abdolreza Daraei

Babol University of Medical Science

Hassan Dastsooz

IIGM: Italian Institute for Genomic Medicine

Yaser Mansoori

Fasa University of Medical Science

\section{Research Article}

Keywords: PGGHG, ODF3B, Carcinoma, Renal Cell

Posted Date: September 22nd, 2021

DOI: https://doi.org/10.21203/rs.3.rs-899664/v1

License: @ (1) This work is licensed under a Creative Commons Attribution 4.0 International License. Read Full License 


\section{Abstract \\ Background}

Renal cell carcinoma (RCC) is the most frequent heterogeneous type of kidney cancer. Although the role of many genes in the development of RCC has been shown, its specific molecular mechanism is still unknown. In this research, we studied the expression profile of two poorly studied genes, $P G G H G$ and $O D F 3 B$, with possible role in renal cancers in our RCC patients' tumors and adjacent normal tissues, as well as their clinicopathological features.

\section{Materials and Methods}

The PGGHG and ODF3B expression was determined using the quantitative real-time PCR in 40 tumor samples and their adjacent non-cancerous tissues. Moreover, Enrichr was used to investigate their pathways, ontologies, and possible upstream transcription factors. Furthermore, STRING was applied to search for interaction between $P G G H G$ and ODF3B.

\section{Results}

The expression level of $P G G H G$ and $O D F 3 B$ was lower in the tumor tissues compared to their adjacent normal tissues. The expression level of $O D F 3 B$ in the patients who had tumor size $>4 \mathrm{~cm}$ was significant. Our data found the possible upstream transcription factor of $O D F 3 B$ and $P G G H G$ regulating their expression in biological pathways, mainly $P P A R G$ as the same upstream transcription factor affecting both genes. Moreover, our data revealed connection between protein networks of $P G G H G$ and $O D F 3 B$. We found several main KEGG, biological process (BP), molecular function (MF), and cellular component (CC) for proteins involved in these networks.

\section{Conclusion}

PGGHG and $O D F 3 B$ downregulation in RCC tumors suggests their possible tumor-suppressive role in RCC. More research is needed to determine the precise mechanism of their involvement in RCC pathogenesis.

\section{Introduction}

Renal cell carcinoma (RCC) is the most common type of kidney malignancies (1) and highly aggressive heterogeneous disease (2). On the basis of data of SEER database, the 5-year relative survival rate of RCC for all SEER stages combined is approximately $75 \%$. The most common histological subtypes of RCC are clear cell, papillary, and chromophobe (3) responsible for $70 \%, 10-15 \%$, and $5 \%$ of the RCC types, respectively (4). So far, many factors have been implicated in the development of CcRCC, including genetic and environmental factors. The majority of ccRCC has shown genetic or epigenetic inactivation of the von Hippel-Lindau (VHL) gene. Moreover, dysregulation of several genes has been contributed to the RCC progression and metastasis in different pathways. CcRCC patients respond poorly to radiotherapy and chemotherapy. Therefore, the molecular predictions of disease progression and metastasis are important for therapies. We are looking to explore new molecular biomarkers of disease progression and ccRCCspecific molecular mechanisms that may provide new targeted treatment options.

This study set out to investigate the potential contribution of two genes with unknown roles, including proteinglucosylgalactosylhydroxylysine glucosidase ( $P G G H G$ ) (also known as ATHL 1) and outer the dense fiber of sperm tails 
3B (ODF3B) (Also known as FAP123; ODF3L3), in RCC pathogenesis. The chromosomal positions of ODF3B and $P G G H G$ genes are $22 q 13.33$ and 11 p15.5, respectively.

The pronephros is consisted of two types of epithelial cells, including transportive and multiciliated cells (MCCs). The epithelial MCCs expresses odf3b protein (5). Previous study showed that odf3b transcripts localize in pronephros cells and labels maturing MCCs in renal progenitors (6). Using STRING database, ODF3B showed interaction with proteins involved in main biological pathways and cancers (including renal cancers), for example WAPAL (7), STAG1 (8), RAD21L1(9) and among others, representing its possible role in these pathways and cancers (Fig. 1).

In relation to the PGGHG, it was found to act in release of glucose from human placental type IV collagen (10). Data extracted from Enrichr (https://maayanlab.cloud/Enrichr/) shows its involvement in hydrolase activity, hydrolyzing 0glycosyl compounds (GO: 0004553). Moreover, previous study conducted by Wei Shi et al. showed higher expression of hsa-mir-484 correlated with worse prognosis in breast cancer. In their study, they found that one of target gene of hsa-mir-484 was $P G G H G(11)$. In addition to these data, its protein network revealed its interaction with several important proteins involved in biological pathways and cancers (including renal cancers), for instance, COL10A1 (12) (Fig. 1). Furthermore, when we constructed and connected the protein network for PGGHG and ODF3B using STRING (https://string-db.org), these two proteins showed involvement in the same network (Fig. 1), suggesting their possible roles in renal pathways and cancers. Therefore, our aim was to investigate their expression and roles in our available samples from renal cancers.

\section{Materials And Methods}

\section{Patient characteristics and tumor samples}

To investigate mRNA expression of $P G G H G$ and $O D F 3 B$ in renal cancers, we collected 40 tumor tissues and their adjacent normal tissues of RCC patients from Ali-Asghar, Namazi, and Ghadir Mother and Child Hospitals (Shiraz, Iran). In total, RCC patients had not received radiotherapy and chemotherapy before surgery. The samples are immediately immersed in liquid nitrogen and stored at $-80^{\circ} \mathrm{C}$ until use. The clinicopathologic and demographic features of RCC patients are shown in Table 1. After surgery, we followed up patients to find they had all-cause mortality. 
Table 1

The association of ODF3B and PGGHG expression levels with demographic and clinicopathological factors in RCC patients.

\begin{tabular}{|c|c|c|c|c|c|c|c|c|c|c|c|}
\hline & & \multicolumn{5}{|c|}{ ODF3B level } & \multicolumn{5}{|c|}{ PGGHG level } \\
\hline & & $\mathbf{N}$ & Mean & SD & Median & $\begin{array}{l}\mathrm{P}- \\
\text { value }\end{array}$ & $\mathbf{N}$ & Mean & SD & Median & $\begin{array}{l}\mathrm{P} \text { - } \\
\text { value }\end{array}$ \\
\hline \multirow[t]{2}{*}{ Sex } & male & 29 & 0.924 & 0.984 & 0.620 & \multirow[t]{2}{*}{0.844} & 29 & 0.795 & 1.037 & 0.309 & \multirow[t]{2}{*}{0.296} \\
\hline & female & 11 & 0.748 & 0.637 & 0.660 & & 11 & 0.971 & 0.857 & 0.798 & \\
\hline \multirow{2}{*}{$\begin{array}{l}\text { Tumor } \\
\text { size }\end{array}$} & $\leq 4$ & 20 & 1.054 & 0.881 & 0.919 & \multirow[t]{2}{*}{0.060} & 20 & 0.758 & 0.843 & 0.540 & \multirow[t]{2}{*}{0.745} \\
\hline & $>4$ & 20 & 0.697 & 0.899 & 0.357 & & 20 & 0.928 & 1.122 & 0.333 & \\
\hline \multirow{2}{*}{$\begin{array}{l}\text { Tumor } \\
\text { focality }\end{array}$} & focal & 25 & 1.097 & 1.031 & 0.774 & \multirow[t]{2}{*}{0.067} & 25 & 0.840 & 1.039 & 0.491 & \multirow[t]{2}{*}{0.856} \\
\hline & unifocal & 15 & 0.507 & 0.434 & 0.587 & & 15 & 0.849 & 0.918 & 0.309 & \\
\hline \multirow{4}{*}{$\begin{array}{l}\text { Tumor } \\
\text { type }\end{array}$} & clear cell & 27 & 0.959 & 0.835 & 0.688 & \multirow[t]{4}{*}{0.004} & 27 & 0.925 & 0.934 & 0.505 & \multirow[t]{4}{*}{0.017} \\
\hline & papillary & 5 & 1.283 & 1.330 & 0.774 & & 5 & 0.974 & 0.958 & 0.644 & \\
\hline & chromophore & 6 & 0.043 & 0.030 & 0.031 & & 6 & 0.069 & 0.096 & 0.029 & \\
\hline & oncocytoma & 2 & 1.231 & 0.864 & 1.231 & & 2 & 1.730 & 2.427 & 1.730 & \\
\hline \multirow{2}{*}{$\begin{array}{l}\text { Tumor } \\
\text { necrosis }\end{array}$} & seen & 20 & 0.924 & 1.086 & 0.601 & \multirow[t]{2}{*}{0.715} & 20 & 0.598 & 0.771 & 0.331 & \multirow[t]{2}{*}{0.160} \\
\hline & not seen & 20 & 0.827 & 0.685 & 0.674 & & 20 & 1.088 & 1.124 & 0.635 & \\
\hline \multirow{4}{*}{$\begin{array}{l}\text { Fuhrman } \\
\text { nuclear } \\
\text { grade }\end{array}$} & 1 & 5 & 0.328 & 0.308 & 0.233 & \multirow[t]{3}{*}{0.113} & 5 & 0.359 & 0.573 & 0.090 & \multirow[t]{3}{*}{0.225} \\
\hline & 2 & 21 & 0.768 & 0.920 & 0.470 & & 21 & 0.818 & 0.961 & 0.399 & \\
\hline & 3 & 9 & 1.189 & 0.711 & 1.603 & & 9 & 1.268 & 1.180 & 0.798 & \\
\hline & 4 & 5 & 1.310 & 1.271 & 1.065 & & 5 & 0.665 & 0.979 & 0.302 & \\
\hline \multirow{2}{*}{$\begin{array}{l}\text { Lymph } \\
\text { vascular } \\
\text { perineural } \\
\text { invasion }\end{array}$} & No & 8 & 1.057 & 1.055 & 0.841 & \multirow[t]{2}{*}{0.467} & 8 & 0.801 & 1.042 & 0.304 & \multirow[t]{2}{*}{0.866} \\
\hline & Yes & 32 & 0.830 & 0.866 & 0.625 & & 32 & 0.854 & 0.985 & 0.445 & \\
\hline \multirow[t]{2}{*}{ Extension } & No & 30 & 0.870 & 0.884 & 0.625 & \multirow[t]{2}{*}{1.000} & 30 & 0.901 & 1.046 & 0.445 & \multirow[t]{2}{*}{0.779} \\
\hline & Yes & 10 & 0.892 & 0.985 & 0.695 & & 10 & 0.670 & 0.787 & 0.330 & \\
\hline \multirow{2}{*}{$\begin{array}{l}\text { Cancer } \\
\text { history }\end{array}$} & No & 19 & 0.891 & 0.998 & 0.587 & \multirow[t]{2}{*}{0.882} & 19 & 0.897 & 1.085 & 0.399 & \multirow[t]{2}{*}{0.903} \\
\hline & Yes & 21 & 0.861 & 0.820 & 0.774 & & 21 & 0.794 & 0.906 & 0.333 & \\
\hline BMI & $\leq 25$ & 9 & 1.224 & 1.433 & 0.616 & 0.918 & 9 & 0.762 & 0.928 & 0.234 & 0.784 \\
\hline & $25-29$ & 26 & 0.771 & 0.689 & 0.645 & & 26 & 0.826 & 1.007 & 0.445 & \\
\hline & $\geq 30$ & 5 & 0.791 & 0.650 & 0.620 & & 5 & 1.080 & 1.134 & 0.798 & \\
\hline Kidney & No & 29 & 0.867 & 0.838 & 0.629 & 0.880 & 29 & 0.880 & 1.038 & 0.357 & 0.940 \\
\hline & Yes & 11 & 0.897 & 1.083 & 0.470 & & 11 & 0.745 & 0.860 & 0.399 & \\
\hline
\end{tabular}




\section{Rna Extraction And Cdna Synthesis}

Using the Trizol isolation reagent (Invitrogen, Thermo Fisher) total RNA was extracted from each sample according to the manufacturer's instructions. Gel electrophoresis and a nanodrop spectrophotometer (BioTek, HTX multi-mode reader) was used to assess RNA quality and quantity, which detected ratios of $\geq 2$ and 1.8 for A260/A230 and A260/A280, respectively. Total RNA was reverse-transcribed into first-strand cDNA using the PrimeScript ${ }^{T M} R^{2}$ Reagent Kit (Takara, Cat.No: RR037A) following manufacturer's recommendations.

\section{Qrt-pcr}

QRT-PCR was carried out using Power SYBR® Green PCR Master Mix (ABI, USA) on the 7500 real-time PCR system (ABI, life technology). $7.5 \mu$ BioFACT ${ }^{\mathrm{TM}}$ master mix including SYBR Green (Ampliqon, Cat.No: A325402-25), along with 1 $\mu \mathrm{l}$ of cDNA, $0.75 \mu \mathrm{l}$ of each primer and $5 \mu \mathrm{l}$ DNase-free $\mathrm{dH} 20$ was used for total volume of $15 \mu \mathrm{l}$ reaction mix. B2M was used as an internal control for normalization of data compared to the studied genes. All reactions were confirmed at least twice and the results were shown to be consistent. The sequences for sense (S) and antisense (AS) primers are shown in Table 2. Relative quantification strategy for quantitative real-time polymerase chain reaction (qPCR) data analysis was calculated by the $2^{-\Delta \Delta C T}$ (fold change) method.

Table 2

Primers sequences

\begin{tabular}{|lll|}
\hline mRNAs and housekeeping gene & Primer & \\
\hline PGGHG & Forward & TCTACTACCTGCTCAGTG \\
\hline ODF3B & Reverse & GTGGAACATCAGGATACT \\
\hline B2M (housekeeping gene) & Forward & GGACCTGGCAGGTACTTC \\
& Reverse & CGTAGATGGAGCAAGTTG \\
\cline { 2 - 3 } & Reverse & GCGGCATCTTCAAACCTCCA \\
\hline
\end{tabular}

\section{Statistical analysis}

Statistical analyses were done in IBM SPSS version 26 software (IBM Corp, Armonk, NY). The data were presented as mean and standard deviation for $\Delta \Delta \mathrm{Ct}$, or median for fold change. Wilcoxon test was used to compare fold changes between tumors and adjacent normal renal tissues. The relation of PGGHG and ODF3B expression with demographic and clinicopathological features was assessed by Mann-Whitney and Kruskal-Wallis tests. In the next step, fold changes were divided into 2 groups of high and low expressions conforming to median for each gene, and the comparison among these groups were analyzed by chi-square test and independent $t$ test. The level of P-value less than 0.05 was regarded as a statistical significance. After surgery, we followed up patients until all-cause death used and censured alive one. The log rank test is a statistical hypothesis test that may be used to compare two high and low gene expression survival curves. 


\section{Bioinformatic Analysis}

We used Enrichr database (https://maayanlab.cloud/Enrichr/) to look for their pathways, ontologies, and possible upstream transcription factors and among others. Moreover, we used STRING (https://string-db.org) to look for interaction between $P G G H G$ and $O D F 3 B$ and their partner, helping identify their possible pathways. To evaluate survival analysis our patients, we used the Kaplan-Meier method based on observed survival times. This analysis was performed using IBM SPSS version 26 software (IBM Corp, Armonk, NY).

\section{Result}

\section{Downregulation of PGGHG and ODF3B gene expression in RCC}

The expression levels of mRNAs were assessed in 40 tumor samples and their tumor's adjacent normal tissues using qRT-PCR. As shown in the Fig. 2A, the expression of $P G G H G$ had significantly lower in tumor tissues (median $=0.378$ ) compared to their adjacent normal tissues (median $=1.120)($ Pvalue $=0.034)$. Also, the expression level of $O D F 3 B$ was significantly lower in tumors tissues (median $=0.625)$ in comparison to the tumor's adjacent normal tissues (median = 0.977) (Pvalue = 0.042, Fig. 2B).

\section{Association between PGGHG and ODF3B gene expression and clinicopathological and demographic features of RCC}

Next, we investigated the relationship between $P G G H G$ and $O D F 3 B$ expression levels and the clinicopathologic status of patients with renal cell carcinoma. Our results demonstrated that ODF3B had lower expression in RCC patients who had tumor size $>4 \mathrm{~cm}$ (Pvalue $=0.060$, Fig. $3 \mathrm{~A}$ ). When we divided fold changes into two high and low expressions groups, chi-square test proved the significant relation between tumor size and ODF3B expression (Pvalue $=0.011$, Table 3). Comparison of $P G G H G$ expression level and tumor size did not show a significant difference $(P v a l u e=0.744)$. 
Table 3

The association of ODF3B and PGGHG expression levels with demographic and clinicopathological factors in RCC patients, according to categorizing patients in to two groups of high and low expressions.

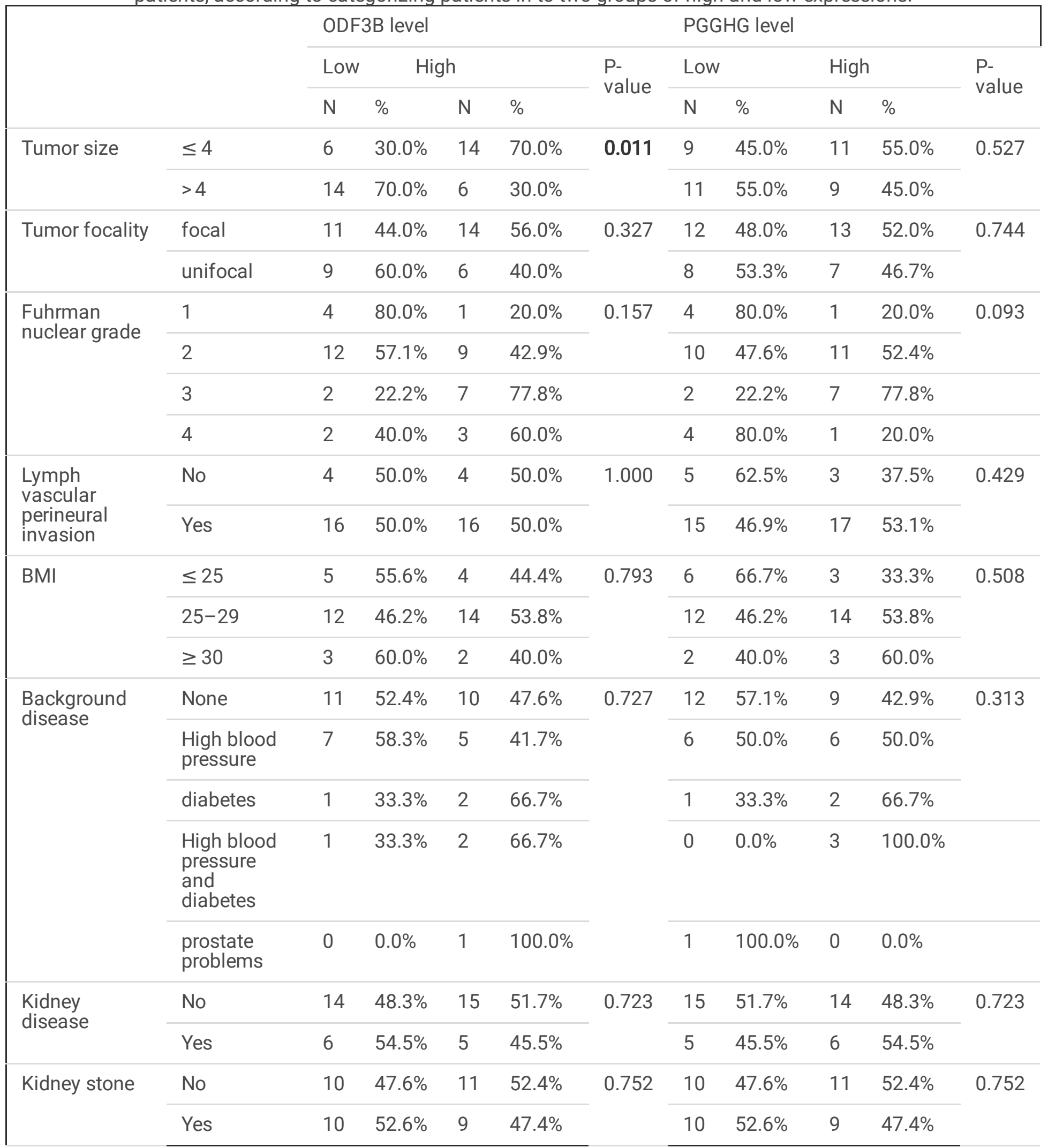

Since ccRCC is the most common of RCC and oncocytoma is the least common type in our study community (Table 1), our analysis showed that PGGHG expression level was higher in oncocytoma type in comparison to other type of RCC 
(Pvalue $=0.017$, Fig. 3B). Similarly, the relationship between the ODF3B expression level and the tumor type showed significant. The expression level of ODF3B was higher in oncocytoma type (Pvalue $=0.004$, Fig. 3C).

\section{Bioinformatic Analysis}

\section{Functional enrichment analysis}

Using Enrichr, we found down-regulation of ODF3B upon RUNX1 Knock-out mouse, PPARG deficiency mouse, SETDB1 Knock-down in THP1 human cells, IRF9 Knock-down in human cells, NANOG over-expressed mouse, and CREM Knockout mouse (Table 4). These data can suggest the possible upstream transcription factors of $O D F 3 B$ which regulate its expression in biological pathways. Among GO ontologies using Enrichr, ODF3B was found to be involved in cytoskeleton (GO: 0005856, p-value: 0.03000 ) in terms of cellular component (CC).

Table 4

Down-regulation of ODF3B mRNA upon transcription perturbations.

\begin{tabular}{|c|c|c|c|c|c|}
\hline Index & Name & P-value & $\begin{array}{l}\text { Adjusted p- } \\
\text { value }\end{array}$ & $\begin{array}{l}\text { Odds } \\
\text { Ratio }\end{array}$ & $\begin{array}{l}\text { Combined } \\
\text { score }\end{array}$ \\
\hline 1 & $\begin{array}{l}\text { RUNX1 KO MOUSE GSE34292 CREEDSID GENE } 2396 \\
\text { DOWN }\end{array}$ & 0.00895 & 0.01826 & 19821 & 93478.06 \\
\hline 2 & $\begin{array}{l}\text { PPARG DEFICIENCY MOUSE GSE23421 CREEDSID } \\
\text { GENE } 1231 \text { DOWN }\end{array}$ & 0.0103 & 0.01826 & 19794 & 90569.85 \\
\hline 3 & $\begin{array}{l}\text { SETDB1 KD THP1 HUMAN GSE103409 SGRNA6DAY } 4 \\
\text { RNASEQ DOWN }\end{array}$ & 0.01105 & 0.01826 & 19779 & 89111.01 \\
\hline 4 & $\begin{array}{l}\text { IRF9 KD HUMAN GSE50588 CREEDSID GENE } 2809 \\
\text { DOWN }\end{array}$ & 0.0132 & 0.01826 & 19736 & 85408.48 \\
\hline 5 & $\begin{array}{l}\text { NANOG OE MOUSE GSE } 48370 \text { CREEDSID GENE } 2980 \\
\text { DOWN }\end{array}$ & 0.01565 & 0.01826 & 19687 & 81844.62 \\
\hline 7 & $\begin{array}{l}\text { CREM KO MOUSE GSE29593 CREEDSID GENE } 2559 \\
\text { DOWN }\end{array}$ & 0.01905 & 0.01905 & 19619 & 77704.89 \\
\hline
\end{tabular}

In relation to the PGGHG, Enrichr revealed its down-regulation upon ZNF503 shRNA in $\mathrm{H} 1$ human cells, NFYC Knock-out in mouse, BCL6 Knock-down in human cells, POU2AF1 over-expression in mouse, EPAS1 Knock-down in HUVEC human cells, NFYA Knock-out in mouse, FOXP3 activation in human cells, PPARG over-expression in mouse, and GATA4 overexpression in A549 human cells (Table 5). 
Table 5

Down-regulation of PGGHG mRNA upon transcription perturbations.

\begin{tabular}{|c|c|c|c|c|c|}
\hline Index & Name & P-value & $\begin{array}{l}\text { Adjusted p- } \\
\text { value }\end{array}$ & $\begin{array}{l}\text { Odds } \\
\text { Ratio }\end{array}$ & $\begin{array}{l}\text { Combined } \\
\text { score }\end{array}$ \\
\hline 1 & $\begin{array}{l}\text { ZNF503 SHRNA H1 HUMAN GSE69618 DAY2 } \\
\text { RNASEQ DOWN }\end{array}$ & 0.00795 & 0.01847 & 19841 & 95923.19 \\
\hline 3 & $\begin{array}{l}\text { NFYC KD MOUSE GSE } 56838 \text { CREEDSID GENE } 1343 \\
\text { DOWN }\end{array}$ & 0.0093 & 0.01847 & 19814 & 92684.96 \\
\hline 5 & $\begin{array}{l}\text { BCL6 KD HUMAN GSE45838 CREEDSID GENE } 1815 \\
\text { DOWN }\end{array}$ & 0.0118 & 0.01847 & 19764 & 87745.54 \\
\hline 6 & $\begin{array}{l}\text { POU2AF1 OE MOUSE GSE12421 CREEDSID GENE } \\
2313 \text { DOWN }\end{array}$ & 0.0122 & 0.01847 & 19756 & 87051.43 \\
\hline 7 & EPAS1 KD HUVEC HUMAN GSE62974 RNASEQ DOWN & 0.0136 & 0.01847 & 19728 & 84784.91 \\
\hline 8 & $\begin{array}{l}\text { NFYA KD MOUSE GSE } 56838 \text { CREEDSID GENE } 1341 \\
\text { DOWN }\end{array}$ & 0.0138 & 0.01847 & 19724 & 84479.77 \\
\hline 9 & $\begin{array}{l}\text { FOXP3 ACTIVATION HUMAN GSE } 41087 \text { CREEDSID } \\
\text { GENE } 1054 \text { DOWN }\end{array}$ & 0.01385 & 0.01847 & 19723 & 84404.16 \\
\hline 10 & $\begin{array}{l}\text { PPARG OE MOUSE GSE10192 CREEDSID GENE } 2733 \\
\text { DOWN }\end{array}$ & 0.0161 & 0.01932 & 19678 & 81249.36 \\
\hline 12 & GATA4 OE A549 HUMAN GSE85001 RNASEQ DOWN & 0.02545 & 0.02545 & 19491 & 71552.36 \\
\hline
\end{tabular}

The interesting result was that while PPARG over-expression in mouse resulted in PGGHG down-regulation, $P P A R G$ deficiency mouse showed $O D F 3 B$ down-regulation. These data may indicate PPARG as the same upstream transcription factor affecting $O D F 3 B$ and $P G G H G$.

Among GO ontologies using Enrichr, PGGHG showed involvement in hydrolase activity, hydrolyzing 0-glycosyl compounds (GO: 0004553, p-value: 0.001850 , in terms of molecular function (MF).

As shown in Fig. 1, protein networks of $P G G H G$ and $O D F 3 B$ are connected and correlated. Therefore, we investigated the $\mathrm{GO}$ terms for all proteins involved in these networks to shed light into the identification of possible pathways of PGGHG and ODF3B.

Enrichr revealed significant KEGG, biological process (BP), MF, and CC for proteins involved in these networks given in Fig. 4. However, the highest significant terms were as follow: KEGG: Starch and sucrose metabolism ( $p$-value: 3.058e43); BP: glucan biosynthetic process (G0:0009250, p-value: 5.259e-24), glycogen biosynthetic process (G0:0005978, pvalue: 5.259e-24), and glycogen metabolic process (G0:0005977, p-value: 8.723e-27); MF: UDP-glucosyltransferase activity (GO:0035251, p-value: 1.319e-8) and 1,4-alpha-oligoglucan phosphorylase activity (G0:0004645, p-value: 2.305e-7); CC: chromosome (G0:0005694, p-value: 1.921e-8). All information and proteins involved in these terms are provided in Supplementary file 1 and 2. These data may propose the possible roles of PGGHG and ODF3B, directly or indirectly, in these GO terms and KEGG pathways.

\section{Overall Survival}


To evaluate survival analysis, we used the Kaplan-Meier method based on observed survival times. However, we were not able to predict the prognosis of renal cell carcinoma patients based on PGGHG (ATHL 1) and ODF3B gene expression using this data (Fig. 5A, 5B). The small proportion of participants evaluated, low duration of follow up and undetected exact cause of death could be some of the reasons.

\section{Discussion}

Renal cell carcinoma (RCC) is the most common form of kidney cancer and accounts for approximately $3.7 \%$ of the total cancer deaths (13). Identification of biomarkers helps in improving the prognosis and early detection of RCC. Despite astonishing molecular advances and their significant impact on the detection of functional mRNAs in malignancies, numerous questions remain regarding molecular interactions and their function and need further attention.

In this study, we compared the PGGHG and ODF3B expression levels in tumor and their adjacent normal tissues in RCC patients and their association with patients' demographical and clinicopathologic factors. Analysis of these biomarkers in tissue samples indicated that they may have value for discriminating RCC patients from healthy controls.

Our results indicate that the expression level of $O D F 3 B$ is significantly lower in tumor tissues than their normal adjacent ones. There are few investigations about the role of the studied genes in the development and progression of cancers. In the case of the $O D F 3 B$ gene, an experimental study related to cancer has not been reported. Jihye Ryu et al. (14) examine promotor activity of $O D F 3 B$ in multiple sclerosis (MS) on large scale by genome-wide association studies (GWAS) signals. In this study, an association of promotor variants with the expression of this gene was reported. In another study, Honglin Zhu et al. (15) showed the ODF3B as potential methylation-regulated differentially expressed gene (DEG). This gene delineates the abnormal activation of immune regulation in the pathogenesis of Systemic sclerosis (SSc).

Our study also showed significant lower expression of PGGHG in RCC tissues in comparison to their adjacent normal specimens. In the past decades, the role of aerobic glycolysis in cancer cells has been discussed, however, data supporting for glycogen biology in RCC are lacking (16). In line with our results, a study demonstrated that reduced $A G L$, a glycogen debranching enzyme, increased tumor growth in patients with bladder cancer by RNA interference screen (17). In another study, AGL loss led to the progression of bladder cancer. Details of this metabolomics pathway showed increased glucose metabolism with the help of serine hydroxymethyltransferase in cells (18).

Our data from Enrichr revealed significant KEGG, MF, BP, and CC for proteins involved in ODF3B and PGGHG networks, suggesting the possible roles of these two proteins, directly or indirectly, in these GO terms and KEGG pathways. Moreover, using Enrichr, we found the down-regulation of $O D F 3 B$ and $P G G H G$ upon perturbations of some transcription factors, mainly PPARG which affected both genes. PPARG, Peroxisome Proliferator Activated Receptor Gamma, is involved in adipocyte differentiation (19) considered a "master regulator" of adipogenesis. Moreover, it represses inflammatory response genes in mouse macrophages (20).

We also identified the positive relationship of tumor size with the ODF3B expression level (Table 3). According to a study by Zhi et al., an increase in tumor size has been reported in the progression and high risk of lymph node metastases (LNM) in CCRCC (21).

In summary, we found lower expression of $O D F 3 B$ and $P G G H G$ in renal cancers and proposed their possible biological pathways and upstream transcription factors. Our results propose investigation of functional studies for $O D F 3 B$ and 
PGGHG genes to identify their exact roles in biological pathways and cancers.

\section{Declarations}

\section{Acknowledgments}

This study was supported by Department of Medical Genetics and Medical biotechnology, Faculty of Medicine, Fasa University of Medical Sciences (FUMS), Fasa, Iran.

Funding: This study was financially supported by 97423 (grant number), Fasa University of Medical Sciences.

Conflict of Interest: The authors declare that there is no conflict of interest.

Availability of data and material:

Samples were provided from Ali-Asghar, Namazi, and Ghadir Mother and Child hospitals, Shiraz, Iran.

\section{Authors' contributions:}

Conceptualization: [Elham Mohammadisoleimani]; Methodology: [Elham Mohammadisoleimani, Zahra Firoozi, and Hassan Dastsooz]; Formal analysis and investigation: [Hassan Dastsooz , Mohammad Mehdi Naghizadeh, and Elham Mohammadisoleimani]; Writing - original draft preparation: [Elham Mohammadisoleimani, Hamed Haghi-Aminjan, Hassan Dastsooz, and Zahra Firoozi]; Writing - review and editing: [Hassan Dastsooz, Abdolreza Daraei, and Yaser Mansoori]; Funding acquisition: [Yaser Mansoori], Resources: [Shahryar Zeighami, Ali Ariafar, Hosein Mansoori]; Supervision: [Yaser Mansoori]. All authors read and approved the manuscript before submission.

\section{Ethics approval:}

The local ethical committee at Fasa University of Medical Sciences (ethical code: IR.FUMS.REC.1398.147) approved this study.

\section{Consent to participate:}

Signed informed consent form was collected from each individual in the patient.

\section{Consent for publication:}

Consent for publication is not required as no identifying personal information is published in this manuscript.

\section{References}

1. Wei W, Lv Y, Gan Z, Zhang Y, Han X, Xu Z. Identification of key genes involved in the metastasis of clear cell renal cell carcinoma. Oncol Lett. 2019;17(5):4321-8.

2. Scoll BJ, Wong YN, Egleston BL, Kunkle DA, Saad IR, Uzzo RG. Age, tumor size and relative survival of patients with localized renal cell carcinoma: a surveillance, epidemiology and end results analysis. J Urol. 2009;181(2):50611.

3. Muglia VF, Prando A. Renal cell carcinoma: histological classification and correlation with imaging findings. Radiol Bras. 2015;48(3):166-74. 
4. Znaor A, Lortet-Tieulent J, Laversanne M, Jemal A, Bray F. International variations and trends in renal cell carcinoma incidence and mortality. Eur Urol. 2015;67(3):519-30.

5. Marra AN, Adeeb BD, Chambers BE, Drummond BE, Ulrich M, Addiego A, et al. Prostaglandin signaling regulates renal multiciliated cell specification and maturation. Proceedings of the National Academy of Sciences of the United States of America. 2019;116(17):8409-18.

6. Marra AN, Cheng CN, Adeeb B, Addiego A, Wesselman HM, Chambers BE, et al. Iroquois transcription factor irx2a is required for multiciliated and transporter cell fate decisions during zebrafish pronephros development. Scientific Reports. 2019;9(1):6454.

7. Stelloh C, Reimer MH, Pulakanti K, Blinka S, Peterson J, Pinello L, et al. The cohesin-associated protein Wapal is required for proper Polycomb-mediated gene silencing. Epigenetics \& chromatin. 2016;9:14-

8. van der Lelij P, Newman JA, Lieb S, Jude J, Katis V, Hoffmann T, et al. STAG1 vulnerabilities for exploiting cohesin synthetic lethality in STAG2-deficient cancers. Life Science Alliance. 2020;3(7):e202000725.

9. Feichtinger J, Aldeailej I, Anderson R, Almutairi M, Almatrafi A, Alsiwiehri N, et al. Meta-analysis of clinical data using human meiotic genes identifies a novel cohort of highly restricted cancer-specific marker genes. Oncotarget. 2012;3(8):843-53.

10. Hamazaki $\mathrm{H}$, Hamazaki MH. Catalytic site of human protein-glucosylgalactosylhydroxylysine glucosidase: Three crucial carboxyl residues were determined by cloning and site-directed mutagenesis. Biochem Biophys Res Commun. 2016;469(3):357-62.

11. Shi W, Dong F, Jiang Y, Lu L, Wang C, Tan J, et al. Construction of prognostic microRNA signature for human invasive breast cancer by integrated analysis. Onco Targets Ther. 2019;12:1979-2010.

12. Chen S, Wei Y, Liu H, Gong Y, Zhou Y, Yang H, et al. Analysis of Collagen type X alpha 1 (COL10A1) expression and prognostic significance in gastric cancer based on bioinformatics. Bioengineered. 2021;12(1):127-37.

13. Chen YY, Hu HH, Wang YN, Liu JR, Liu HJ, Liu JL, et al. Metabolomics in renal cell carcinoma: From biomarker identification to pathomechanism insights. Arch Biochem Biophys. 2020;695:108623.

14. Ryu J, Woo J, Shin J, Ryoo H, Kim Y, Lee C. Profile of differential promoter activity by nucleotide substitution at GWAS signals for multiple sclerosis. Medicine (Baltimore). 2014;93(28):e281.

15. Zhu H, Zhu C, Mi W, Chen T, Zhao H, Zuo X, et al. Integration of Genome-Wide DNA Methylation and Transcription Uncovered Aberrant Methylation-Regulated Genes and Pathways in the Peripheral Blood Mononuclear Cells of Systemic Sclerosis. Int J Rheumatol. 2018;2018:7342472.

16. Xie H, Song J, Godfrey J, Riscal R, Skuli N, Nissim I, et al. Glycogen metabolism is dispensable for tumour progression in clear cell renal cell carcinoma. Nat Metab. 2021;3(3):327-36.

17. Guin S, Pollard C, Ru Y, Ritterson Lew C, Duex JE, Dancik G, et al. Role in tumor growth of a glycogen debranching enzyme lost in glycogen storage disease. J Natl Cancer Inst. 2014;106(5).

18. Weinhaus B, Guin S. Involvement of glycogen debranching enzyme in bladder cancer. Biomed Rep. 2017;6(6):5958.

19. Evans RM, Barish GD, Wang Y-X. PPARs and the complex journey to obesity. Nature Medicine. 2004;10(4):355-61.

20. Pascual G, Fong AL, Ogawa S, Gamliel A, Li AC, Perissi V, et al. A SUMOylation-dependent pathway mediates transrepression of inflammatory response genes by PPAR-gamma. Nature. 2005;437(7059):759-63.

21. Zhi Y, Li X, Qi F, Hu X, Xu W. Association of Tumor Size with Risk of Lymph Node Metastasis in Clear Cell Renal Cell Carcinoma: A Population-Based Study. J Oncol. 2020;2020:8887782.

\section{Figures}

Page $12 / 17$ 


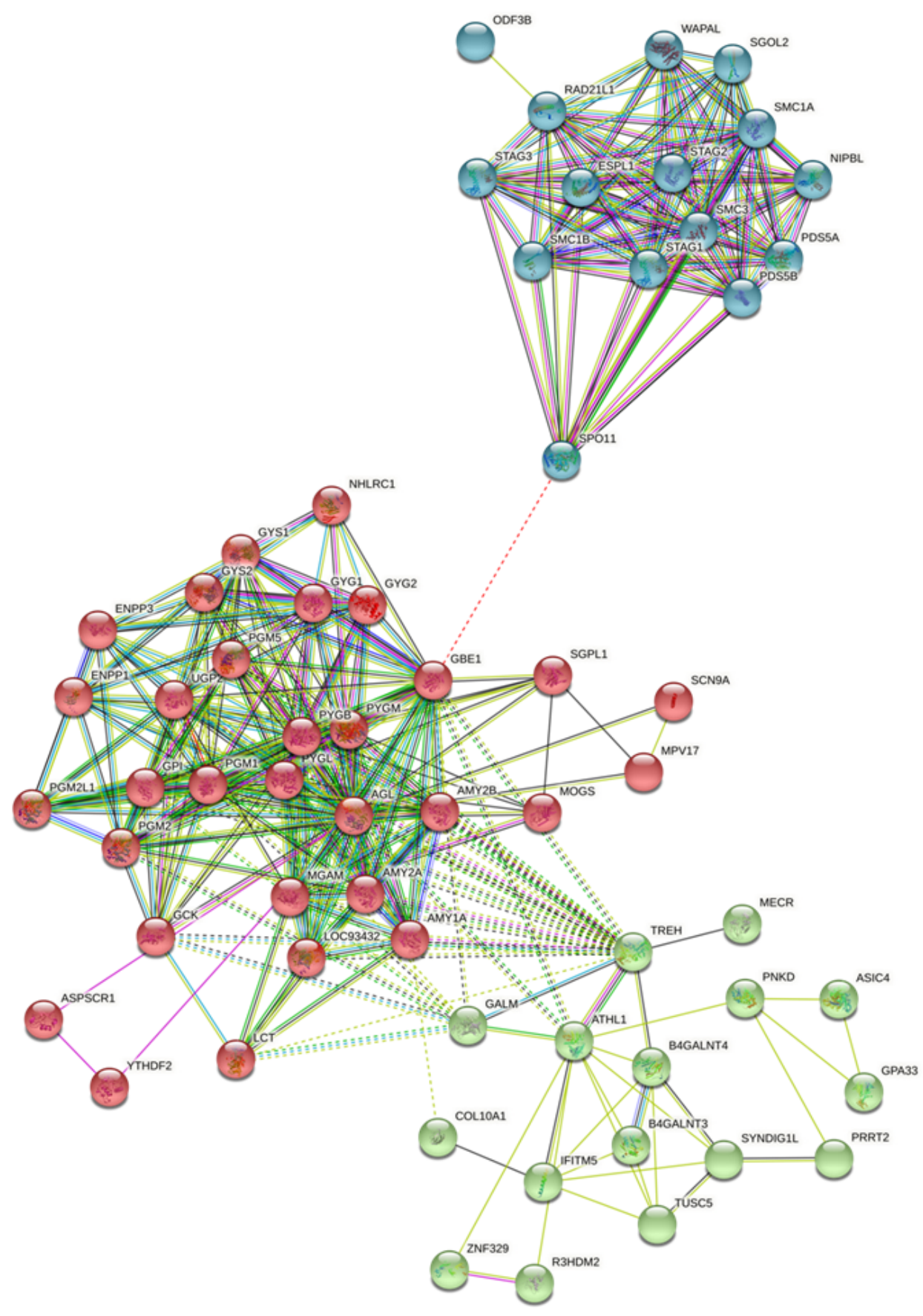

Figure 1

Protein-protein interaction network of PGGHG and ODF3B. It shows connection between protein networks of PGGHG and ODF3B, representing their possible roles in the same pathways and cancer pathogenesis 

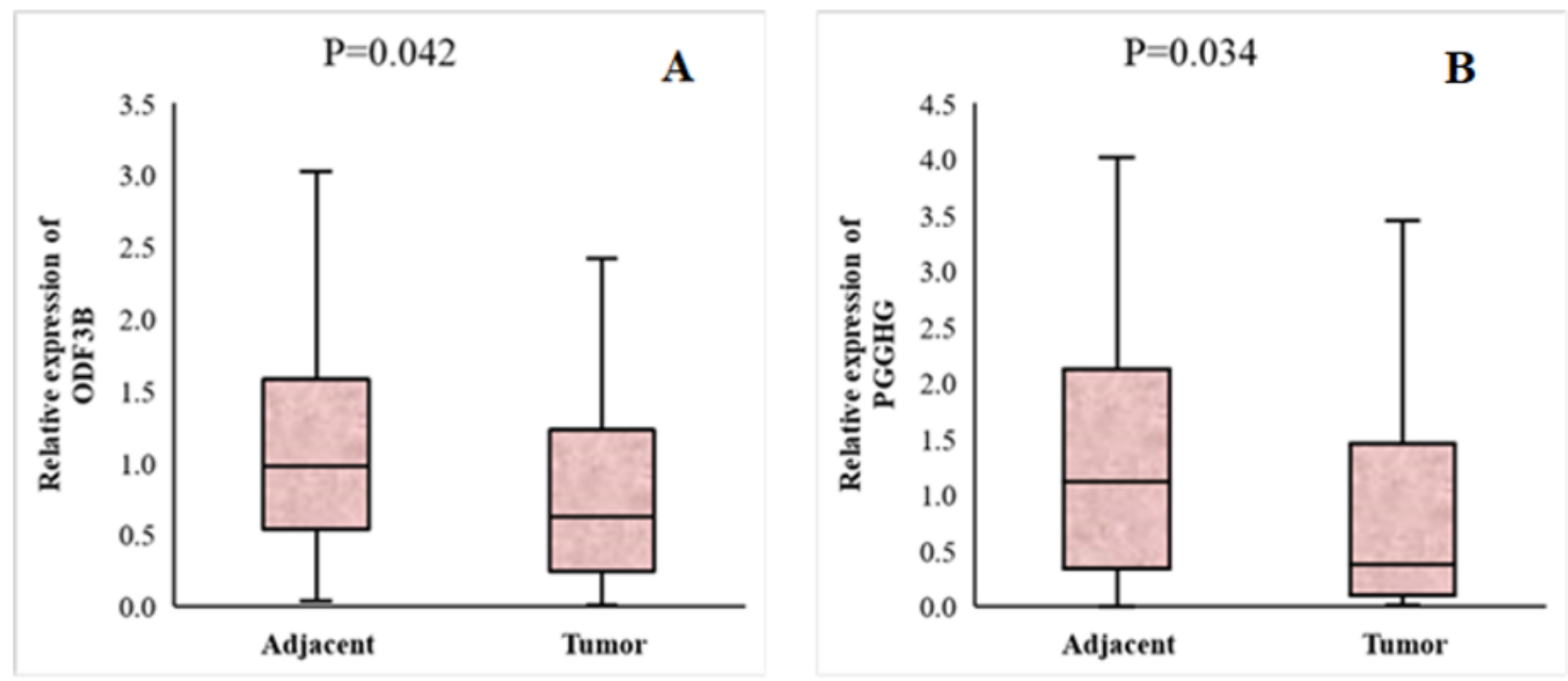

Figure 2

The boxplot of comparison of PGGHG and ODF3B expression between tumor samples and paired adjacent normal tissues 

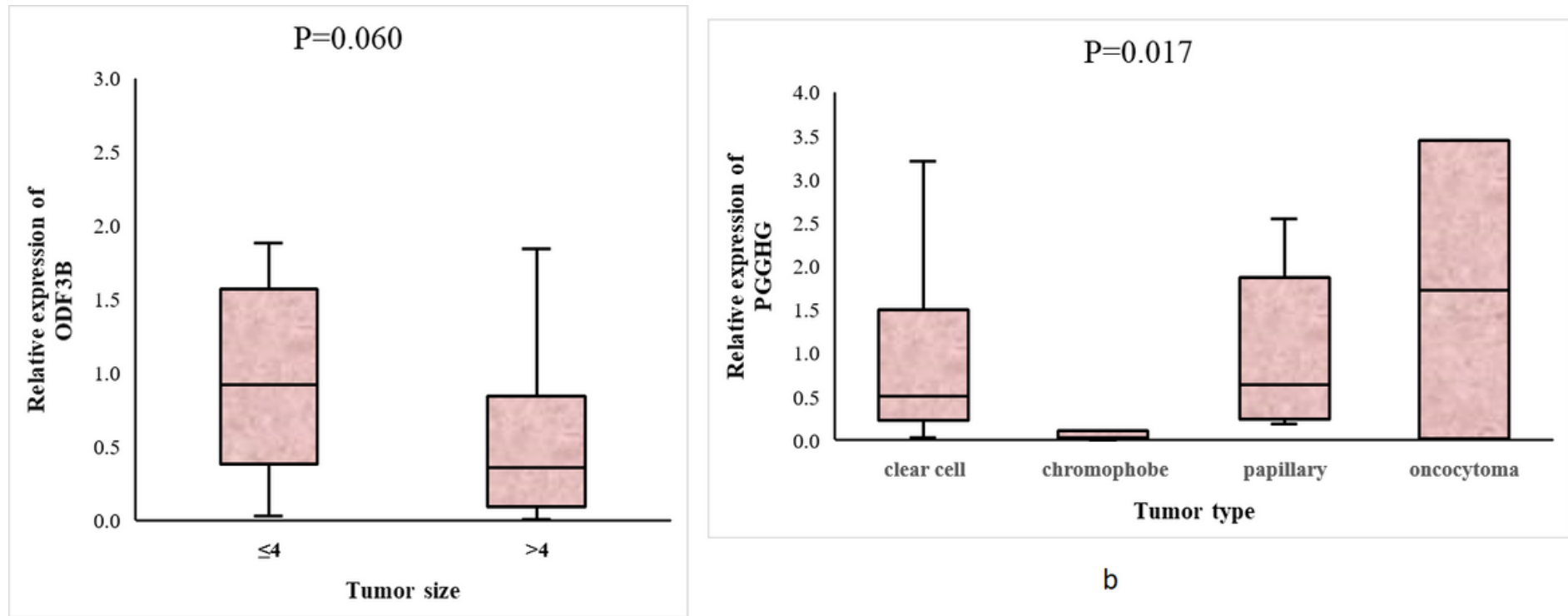

a

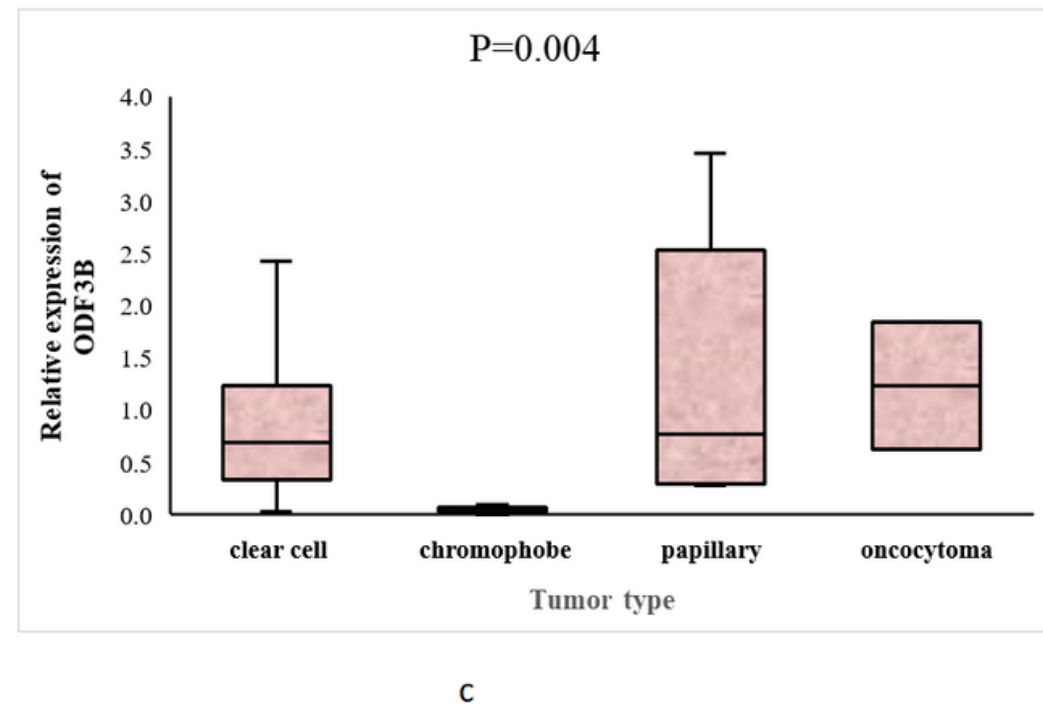

Figure 3

A) The association between the expression of ODF3B and tumor size. B,C) PGGHG and ODF3B expression levels in the different subgroups of tumor types in RCC 


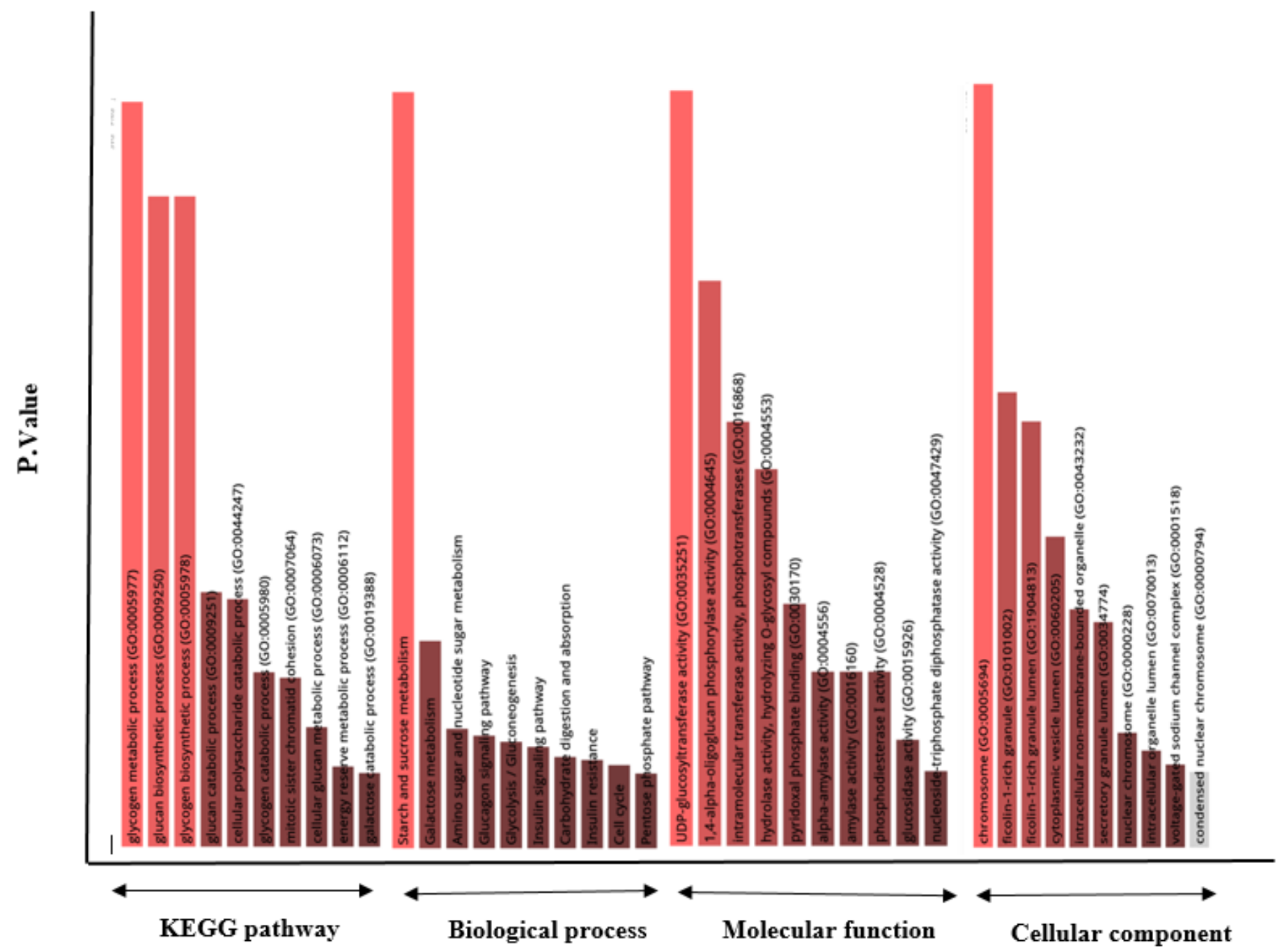

Figure 4

Significant KEGG (Kyoto Encyclopedia of Genes and Genomes) human pathways, BP (biological process), MF (molecular function), and CC (cellular component) GO terms for proteins involved in PGGHG and ODF3B networks 


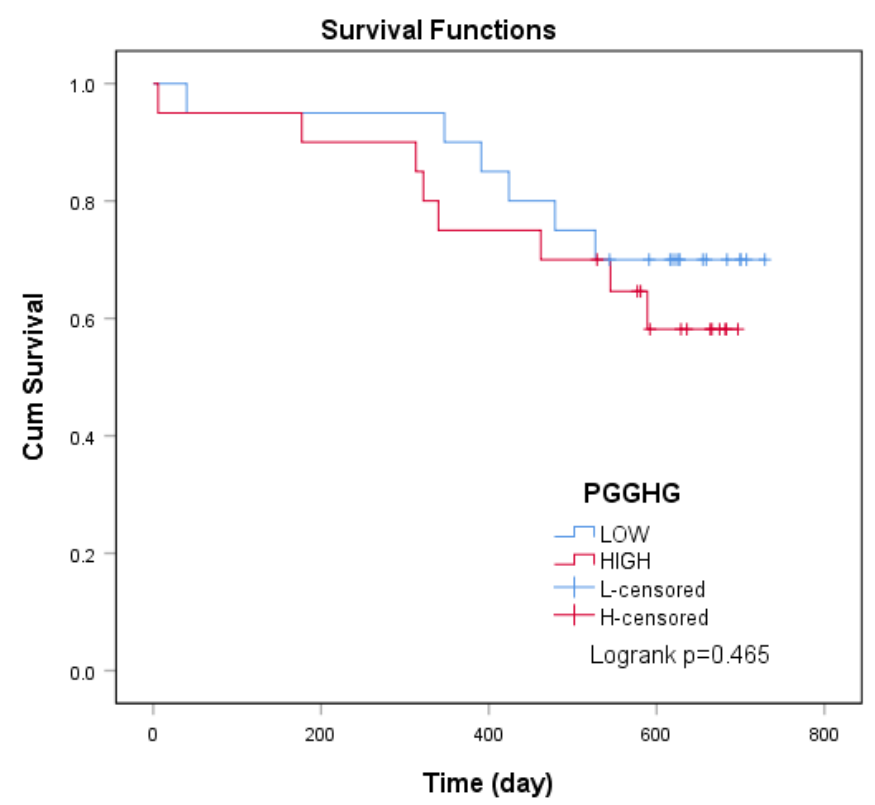

a

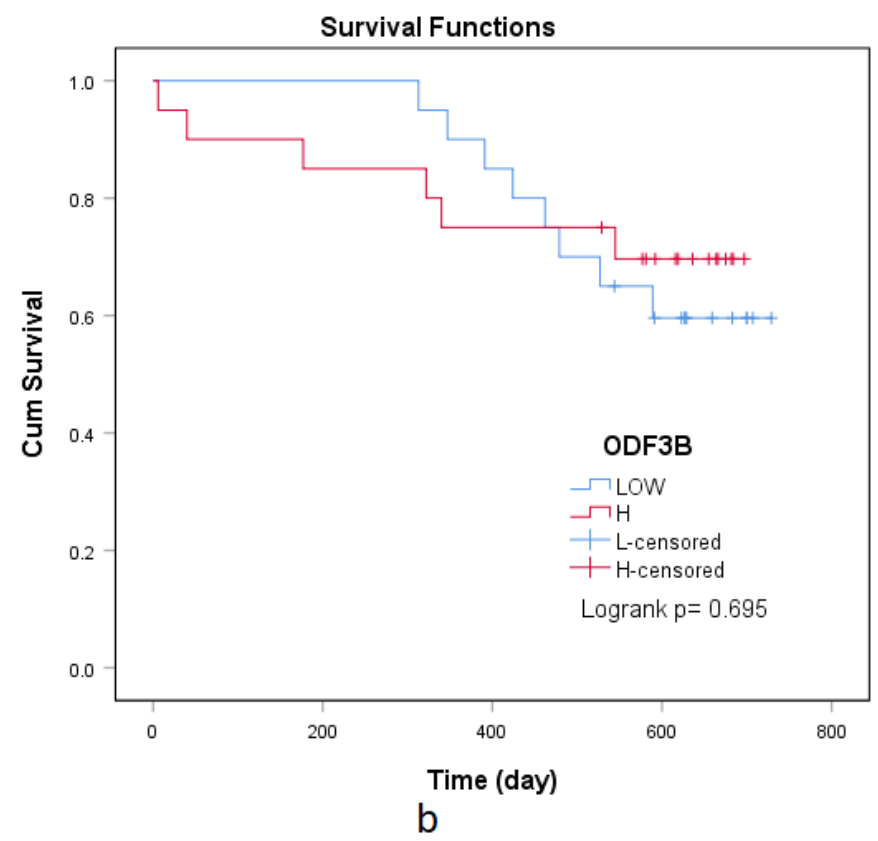

Figure 5

Kaplan-Meier curves for survival rate. Overall survival of patients by 2-year (A: PGGHG, B: ODF3B)

\section{Supplementary Files}

This is a list of supplementary files associated with this preprint. Click to download.

- SupplementaryFiles.rar 